\title{
Cognitive Symbiotic Network Planning for Energy Consumption Reduction in Wireless Sensor Networks
}

\author{
D. Plets*, W. Joseph, E. De Poorter, L. Martens, I. Moerman \\ Department of Information Technology, Ghent University/IBBT \\ Gaston Crommenlaan 8, B-9050 Ghent, Belgium \\ david.plets@intec.ugent.be
}

\begin{abstract}
The concept of cognitive symbiotic network planning is introduced as a way to improve the use of available resources and infrastructure and the overall performance of co-located wireless networks. A real-time network planner is designed that jointly optimizes different networks that cooperate based on shared incentives. Feedback about the signal quality parameters allows monitoring of the network, optimizing path loss models, and coping with a changing propagation environment. The concept is applied to and validated with a real-life wireless test environment for the specific incentive of an energy consumption reduction. A global reduction of more than $75 \%$ is obtained.
\end{abstract}

\section{INTRODUCTION}

In recent years, an increasing number of networks using different wireless technologies started to co-exist: GSM (Global System for Mobile Communications), UMTS (Universal Mobile Telecommunications System), Zigbee, Wi-Fi, LTE (Long Term Evolution),... Fig. 1 shows a ground plan of an office building, containing a Zigbee sensor network (sensors indicated with circles) and a WiFi access point network. In the future, also (LTE) femtocells might start to appear in large buildings. Moreover, most current smartphones and laptops are Bluetooth-compatible as well. This leads to a huge amount of different co-existing wireless technologies in indoor environments. The increase in the density of wireless devices, each using their own technology and having their own limitations (battery lifetime, memory capacity,...) leads to an increasing amount of interference and a sub-optimal use of available network resources (such as capacity).

In this paper, the concept of cognitive symbiotic network planning is developed. The network planner automatically designs a symbiotic network out of a set of co-located independent wireless networks. Symbiotic networks [1] are coexisting homogeneous (using the same technology, e.g., two independent Wi-Fi networks from different companies, sharing their access points) or heterogeneous (e.g., Wi-Fi and UMTS networks allowing a smartphone user to collect data) networks that cooperate based on common incentives through infrastructure and resource sharing. Symbiotic networks form a promising concept in a world where a lot of wireless technologies coexist independently from each other. Incentives are network goals that should be improved in order for the network to consider cooperating with other networks. The aim of the cooperation is to provide benefits for all participating

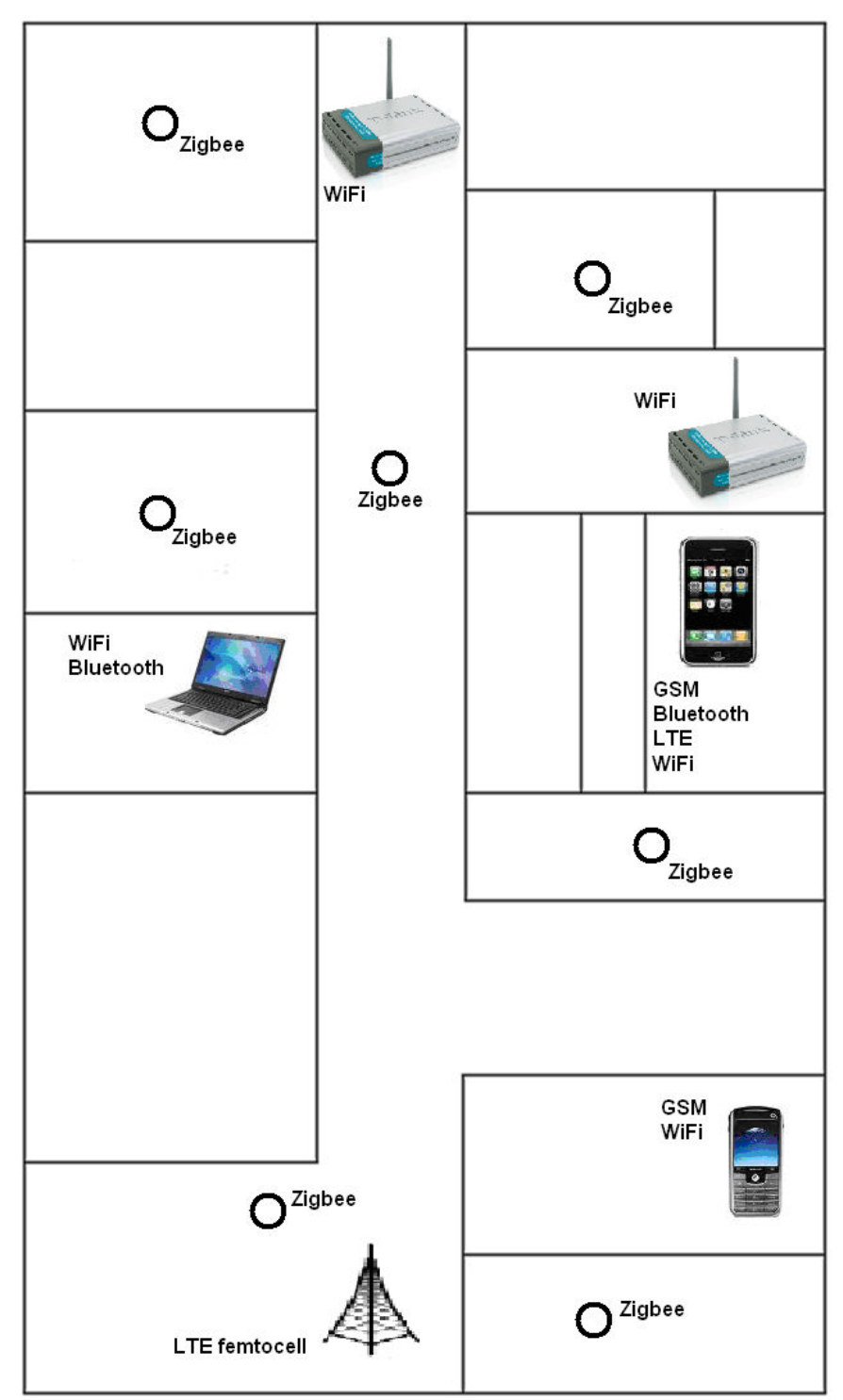

Fig. 1. Ground plan of building with different co-existing wireless technologies. 


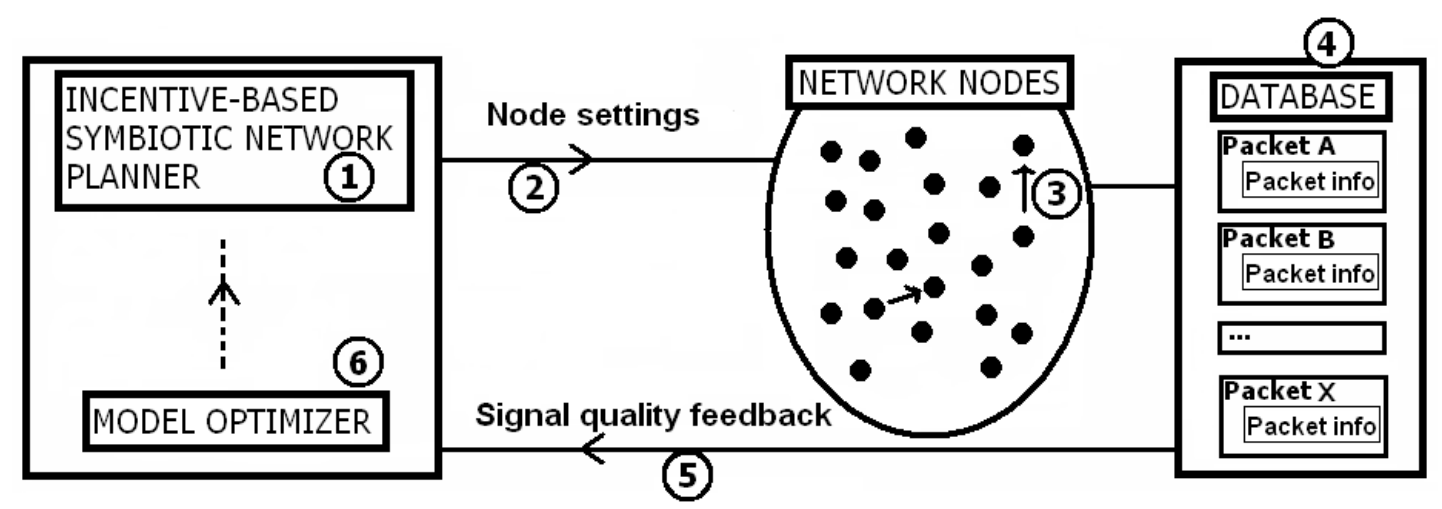

Fig. 2. Flow graph of interaction between cognitive symbiotic network planner and network.

networks. Possible incentives to support cooperation between the different networks are lower exposure, lower interference, a higher throughput, a better QoS (Quality of Service),... These incentives can vary in the course of time.

In this paper, a wireless sensor network will be symbiotically optimized, based on the shared incentive between the networks of a lower energy consumption (for an increased device and network life time).

However, while striving to meet each one of these incentives, it is crucial that the network remains operational. Therefore, we choose to implement the symbiotic networking concept into a cognitive indoor network planning tool. The proposed solution introduces a feedback loop between the planning tool and the actual deployed network. Feeding back signal quality information allows to better tune the device transmit powers for a further energy consumption reduction, but also has other advantages.

As an application, the concept of a cognitive symbiotic network planner is applied to a real-life wireless test environment, where we aim to reduce the global energy consumption, without affecting coverage.

\section{Cognitive Symbiotic Network Planning}

The cognitive symbiotic network planner is illustrated in Fig. 2. Based on the agreed common incentives, the symbiotic network planning algorithm [2] first calculates the optimal node settings (e.g., transmit power) (step 1 in Fig. 2) and applies them to the network nodes (step 2 in Fig. 2). Based on the calculated node settings, the network is reconfigured, packets are sent accordingly (step 3 in Fig. 2), and transmission information from the receiver nodes (Received Signal Strength Indicator (RSSI), noise floor,... ) is logged in a database (step 4 in Fig. 2).

In view of better meeting the agreed incentives and still keeping the network operational, a cognitive loop is implemented into the network planner: signal quality data are returned from the database to the network planner (step 5 in Fig. 2) and used to recalculate the optimal network settings for a further energy consumption reduction (step 6 in Fig. 2). Also, by monitoring the network, real-time network and signal quality information can be used in the planning tool to increase the accuracy of the used propagation models [2], or to adapt to varying network conditions (e.g., node failures) or a varying propagation environment.

Firstly, based on the observed RSSI values, the used path loss models can be tuned to cope with prediction inaccuracies [2] (feedback loop in Fig. 2). This allows a more reliable estimation of the connection quality and possibly, an optimization of the transmit power of the nodes (increased node lifetime). Secondly, the self-regulating process is able to automatically deal with changes in the physical network layout. This for example means that depending on the specific case and on the network topology, it allows detecting node failures and recovering from it. Also, our symbiotic planning tool allows coping with a varying propagation environment. This could e.g., be the case in office buildings with movable walls, where temperature sensors monitor the environment. A rearrangement of the walls in the office building would then not require a manual adjustment of the network parameters, but it could be dealt with by the self-regulating network planner. In a more general approach, the network planner can also automatically be reconfigured when the shared incentives change. When the temparature sensors record values higher than a certain treshold (indication of possible fire), the incentive can change from minimal energy consumption to highly-reliable communication in emergency cases, in order to detect and localize the fire as quickly as possible.

\section{ENERGY CONSUMPTION REDUCTION By COGNITIVE Symbiotic Network Planning in a Real-Life WIRELESS TEST NETWORK}

As a proof-of-concept, the cognitive symbiotic network planner is applied to a real-life wireless test network. In total, 45 nodes, equipped with $2 \mathrm{Wi}$-Fi IEEE 802.11 interfaces (a/b/g) and 1 or 2 TMoteSky sensor nodes [3] with IEEE 802.15.4 interface embedded with temperature, light, and humidity sensors, have been installed at a height of $2.5 \mathrm{~m}$ on the third floor of an office building in Ghent, Belgium (see Fig. 3). The sensor chip is an RF (Radio Frequency) transceiver designed for low-power and low-voltage wireless applications 


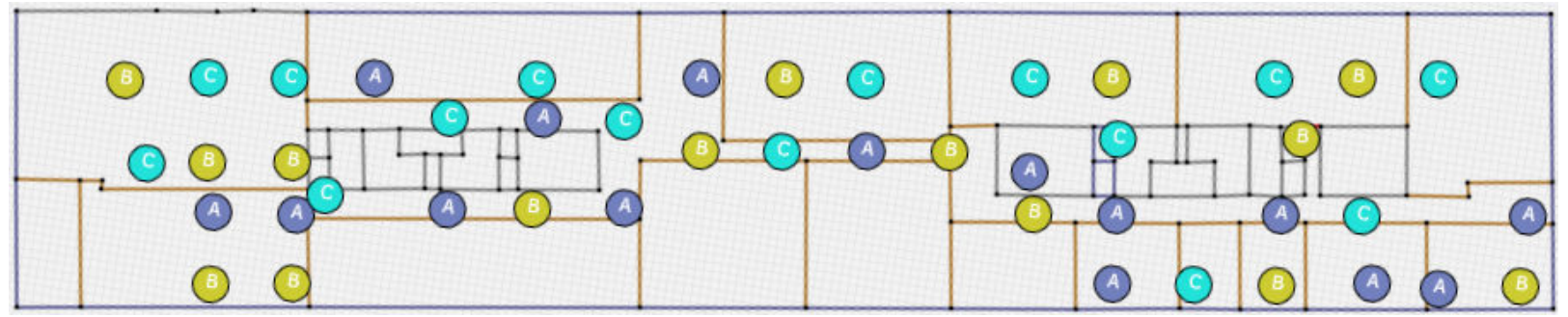

Fig. 3. Original test network nodes from three networks (A, B, C).

and has a programmable output power [3]. In receiving mode, the Received Signal Strength Indication (RSSI) indicates the received power and is a good indicator for the packet reception rate (PRR) when the noise is limited [4].

The 45 nodes of the wireless test network belong to three different sensor networks: A, B, and C (see Fig. 3). Four network configuration phases are defined, corresponding with three subsequent optimizations. The specific common incentive of a lower global energy consumption in the three networks is investigated. It will be shown that the use of a network planner, the use of symbiotic negotiation between the networks, and the use of the cognitive loop all allow a reduction of the global energy consumption in the wireless sensor network.

phase 1: initially, all 45 nodes are 'on', each of which consumes $65.01 \mathrm{~mW}$, yielding a resulting total power consumption of $45 \cdot 65.01 \mathrm{~mW}$ or $2925.5 \mathrm{~mW}$. Fig. 3 shows this original network. In this first phase, no intelligence is used.

phase 2: in the second phase, the network planner optimizes each of the three wireless sensor networks of Fig. 3 separately. In Fig. 4, the resulting sinks are indicated with a circle: 3 for network A, 4 for network $\mathrm{B}$, and 4 for network $\mathrm{C}$. Together, these eleven sinks consume $11 \cdot 65.01 \mathrm{~mW}$ or $715.1 \mathrm{~mW}$. The other nodes are assumed to be 'on' only during a certain percentage of the time, allowing them to send packets and/or receive incoming packets. Under the assumption of a duty cycle of $25 \%$, the other 34 sensor nodes consume a power of $34 \cdot 65.01 \mathrm{~mW} \cdot 25 \%$ or $552.6 \mathrm{~mW}$. For all 45 nodes, this results in a total power consumption of $715.1 \mathrm{~mW}+$ $552.6 \mathrm{~mW}=1267.7 \mathrm{~mW}$.

phase 3: in the third phase, the three networks cooperate in order to consume less energy (i.e. the shared incentive). After a negotiation phase based on higher-layer protocols, the three networks form one symbiotic network, in which the different networks share their infrastructure. The incentive-based network planner is now able to calculate the number of sinks which are required to establish a reliable communication. In Fig. 4, these four sinks are indicated with a square, consuming a power of 4 . $65.01 \mathrm{~mW}$ or $260.4 \mathrm{~mW}$. With a duty cycle of $25 \%$, the other 41 sensor nodes consume a power of $41 \cdot 65.01 \mathrm{~mW}$ . $25 \%$ or $666.4 \mathrm{~mW}$. The total power consumption is then
$260.4 \mathrm{~mW}+666.4 \mathrm{~mW}=926.8 \mathrm{~mW}$.

- phase 4: in the fourth phase, another advantage of the cognitive loop comes into play: the loop not only allows the network planner to improve its path loss models [2], it also allows optimizing the individual sensor transmit powers, using the path loss values fed back to the tool by the cognitive loop. Based on these path loss values, the transmit powers of the sensor nodes are configured at different values, ranging from $-15 \mathrm{dBm}$ to $0 \mathrm{dBm}$.

Table I shows the power consumption corresponding to the different sensor transmit powers, the number of sensors in the network with that transmit power (as decided by the network planner), and the total power consumption of the receiving nodes, with an assumed duty cycle of $25 \%$. The table shows that relevant energy savings can be obtained by not always transmitting at the maximal power of $0 \mathrm{dBm}$ when this is not necessary: an energy reduction of $43 \%$ is obtained when transmitting at $-15 \mathrm{dBm}$ instead of at $0 \mathrm{dBm}$ (power consumption of $57.4 \mathrm{~mW}$ at $0 \mathrm{dBm}$ vs. $32.7 \mathrm{~mW}$ at $-15 \mathrm{dBm}$ ). The total power consumption of all nodes in the network then becomes $260.4 \mathrm{~mW}$ (4 sinks) $+425.8 \mathrm{~mW}$ (41 receiving nodes, see Table I) $=686.2 \mathrm{~mW}$.

\begin{tabular}{|r|r|r|r|}
\hline $\begin{array}{r}\text { sensor } \\
\text { transmit } \\
\text { power }[\mathbf{d B m}]\end{array}$ & $\begin{array}{r}\text { power } \\
\text { consumption } \\
{[\mathbf{m W}]}\end{array}$ & $\begin{array}{r}\text { number of } \\
\text { sensors in } \\
\text { network }\end{array}$ & $\begin{array}{r}\text { total power } \\
\text { consumption [mW] } \\
\text { (duty cycle 25\%) }\end{array}$ \\
\hline 0 & 57.4 & 8 & 114.8 \\
\hline-5 & 46.2 & 9 & 104.0 \\
\hline-10 & 36.3 & 12 & 108.9 \\
\hline-15 & 32.7 & 12 & 98.1 \\
\hline \multicolumn{2}{|c|}{ Total power consumption of receiving nodes } \\
\hline
\end{tabular}

TABLE I

OVERVIEW OF POWER CONSUMPTION FOR DIFFERENT SENSOR TRANSMIT POWERS AND TOTAL POWER CONSUMPTION OF ALL RECEIVING NODES IN OPTIMIZATION PHASE 4.

Table II shows the power consumption (reduction) in the different network configuration phases. It shows that a total energy consumption reduction of $\mathbf{7 6 . 5 \%}$ can be obtained for the network under test. Other duty cycle values will increase or decrease the obtained energy consumption reduction. 


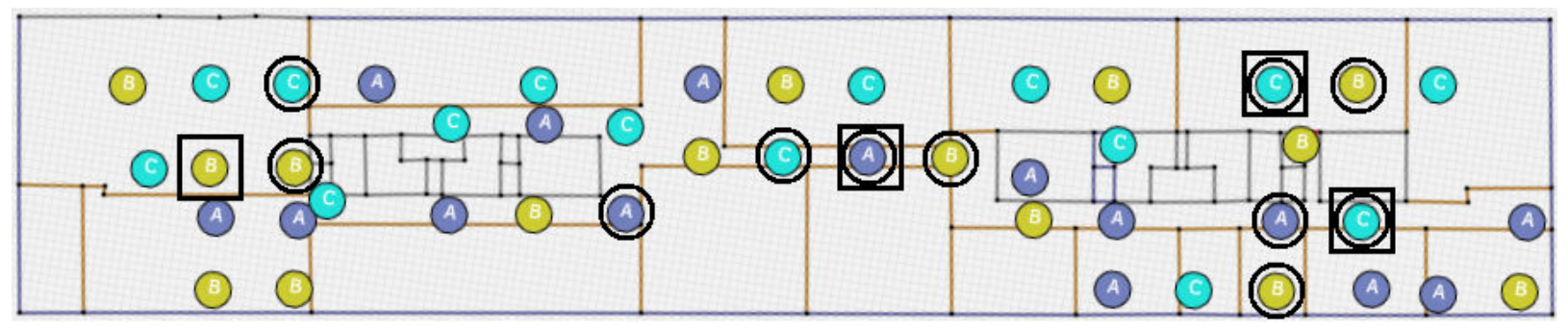

Fig. 4. Indication of sinks for each of the three networks (circle) and for the symbiotic network (squares).

\begin{tabular}{|c|r|r|}
\hline & $\begin{array}{r}\text { total power } \\
\text { consumption [mW] }\end{array}$ & $\begin{array}{r}\text { energy consumption } \\
\text { reduction [\%] }\end{array}$ \\
\hline \hline phase 1 & 2925.5 & - \\
\hline phase 2 & 1267.7 & $56.7 \%$ \\
\hline phase 3 & 926.8 & $68.3 \%$ \\
\hline phase 4 & 686.2 & $76.5 \%$ \\
\hline
\end{tabular}

TABLE II

OVERVIEW OF THE POWER CONSUMPTION (REDUCTION) IN THE DIFFERENT NETWORK CONFIGURATION PHASES.
[2] D. Plets, W. Joseph, K. Vanhecke, and L. Martens, “Automatic Network Optimization and Dynamic Network Management Using a Propagation Prediction Tool in a Living Lab Setting," in European Conference on Antennas and Propagation 2011, Rome, 11-15 April 2011, paper no. 1569369977.

[3] Texas Instruments, "2.4 GHz IEEE 802.15.4 / ZigBee-ready RF Transceiver Datasheet," Tech. Rep. [Online]. Available: http://www.ti.com/lit/gps/cc2420

[4] K. Srinivasan and P. Levis, "RSSI Is Under-Appreciated," in Proceedings of the Third Workshop on Embedded Networked Sensors (EmNets), Cambridge, MA, May 2006.

\section{CONCLUSIONS}

The concept of a cognitive symbiotic network planning tool is presented. It creates an optimized incentive-based symbiotic network starting from different independent wireless networks. This allows to e.g., lower the global energy consumption of the co-located wireless networks. A cognitive loop is implemented into the network planning tool, enabling the automatic optimization of node transmission settings for a further energy consumption reduction. Also, the loop guarantees a better overall performance and allows dealing with changes in the propagation environment or in the physical network layout. In this way, a a total energy consumption reduction of more than $75 \%$ can be obtained. Future research includes incentive-based network optimization in heterogeneous symbiotic networks, e.g., the joint optimization of LTE and WiFi networks for a lower exposure, lower energy consumption or a better coverage.

\section{ACKNOWLEDGMENT}

This work was supported by the IBBT-SBO SymbioNets project, co-funded by the IBBT (Interdisciplinary institute for BroadBand Technology), a research institute founded by the Flemish Government in 2004, and the involved companies and institutions. W. Joseph is a Post-Doctoral Fellow of the FWO$\mathrm{V}$ (Research Foundation-Flanders).

\section{REFERENCES}

[1] E. D. Poorter, B. Latre, I. Moerman, and P. Demeester, "Symbiotic networks: Towards a new level of cooperation between wireless networks," Special Issue of the Wireless Personal Communications Journal, vol. 45, no. 4, pp. 479-495, June 2008. 